\title{
Effect of Gestational Age, Postnatal Age, and Illness on Plasma Cortisol Concentrations in Premature Infants
}

\author{
SUSAN M. SCOTT AND KRISTI L. WATTERBERG
}

Department of Pediatrics, Children's Hospital of New Mexico, University of New Mexico School of Medicine, Albuquerque, New Mexico 87123-5311 [S.M.S.], and Department of Pediatrics, Pennsylvania State University Hospital, Milton S. Hershey Medical Center, Hershey, Pennsylvania 17033 [K.L.W.] \begin{abstract}
ABS
The purpose of this study was to define the pattern of post-
natal plasma cortisol concentrations during the first week of life in premature infants, and to evaluate the effect of developmental and clinical factors on this pattern. We measured plasma cortisol concentrations in the morning and afternoon on $\mathrm{d} 2,4$, and 6 in 120 premature infants (gestational age $24-36 \mathrm{wk}$ ) and examined the effects of gestational age, postnatal age, and illness. We described an inverse relationship between gestational age and cortisol concentrations, with the youngest infants having the highest random cortisol values ( $F=5.14, p=0.0073$ ). Illness had a significant negative effect such that the cofactors ventilatory support pattern $(F=6.62, p=0.0016)$ or "use of surfactant" $(F=6.63, p=0.001)$ defined a pattern where cortisol values were lower in infants that had the highest ventilatory requirements or that received surfactant compared with values from those infants who did not have these requirements. The postnatal pattern in cortisol values depended on gestational age.
\end{abstract}

Ill infants more than 27 wk gestational age increased their cortisol values from $\mathrm{d} 2$ to $\mathrm{d} 6$ although cortisol values decreased in well infants. These patterns resulted in a nonsignificant change over time for these age groups. In contrast, cortisol values significantly decreased from $\mathrm{d} 2$ to $\mathrm{d} 6$ in both well and ill infants that were less than or equal to $27 \mathrm{wk}$. We conclude that plasma cortisol concentrations in the premature infant are significantly correlated with gestational age and to markers of illness. Interpretation of the physiologic significance of random cortisol values in this patient population must be made with consideration for the statistically significant effects described above. (Pediatr Res 37: 112-116, 1995)
Abbreviations
ELBW, extremely low birth weight
CBG, cortisol-binding globulin
RAS, respiratory acuity score

Recent studies have reported an association between low random cortisol values, clinical signs consistent with adrenal insufficiency, and resolution of these findings with cortisol supplementation in the ELBW infant $(1,2)$. The authors of these studies suggested that the ELBW infant is at increased risk for adrenal insufficiency; however, there is limited information for cortisol values in these infants. Doerr et al. (3) reported that the well infant of more than $32 \mathrm{wk}$ gestation has a significant decrease in cortisol from birth and that values at $7 \mathrm{~d}$ are similar to those of full-term infants. This information is not available for more immature or sick premature infants.

Metzger et al. (4) recently reported in a small number of premature infants in whom the ultradian (pulsatility within

Received March 14, 1994; accepted March 22, 1994.

Correspondence: Dr. Susan M. Scott, University of New Mexico, Department of Pediatrics, Acc 3-West, Albuquerque, NM 87123-5311.

Supported in part by the Bristol-Myers Research Grant, the General Clinical Research Center of the University of New Mexico, Program DRR (National Institutes of Health 5M01RR00997-14-18), and by Grant MCJ-420627 from the Maternal and Child Health Bureau, Health Resources and Service Administration, Department of Health and Human Services. 24-h cycle) pattern of plasma cortisol concentrations had a higher baseline and lower amplitude than that seen in the full-term infant. That study and the report by Hanna et al. (5) that the ill premature infant has elevated corticotropinreleasing hormone concentrations in comparison with the adult raise concerns about the adequacy of the hypothalamicpituitary-adrenal axis in the ELBW infant, in particular during periods of illness.

The purpose of this study was to define the pattern of postnatal plasma cortisol concentrations during the first week of life in premature infants and to evaluate the effect of developmental and clinical factors on this pattern.

\section{METHODS}

Subjects. All appropriate for gestational age infants less than 37 wk gestational age admitted to the newborn intensive care unit at the Children's Hospital of New Mexico were eligible for the study. No infants were of diabetic mothers, nor were they large or small for gestational age. Infants were enrolled after 
informed consent was obtained from the parents or guardians. To avoid the effects of the cortisol surge at birth on the postnatal cortisol pattern, blood samples for cortisol measurements were collected in the morning and afternoon of $\mathrm{d} 2,4$, and $6(0.1 \mathrm{~mL}$ per sample). Morning samples on $\mathrm{d} 2$ and $6(0.3$ $\mathrm{mL} / \mathrm{sample}$ ) were also analyzed for $\mathrm{CBG}$ to examine the relationship of cortisol to $\mathrm{CBG}$.

Baseline information obtained on each infant included gestational age, sex, and use of exogenous surfactant. Clinical data collected on the same days as the blood samples included "use of inotropes" and maximum ventilator settings each day. The magnitude of respiratory support was estimated with RAS, a clinical score shown to correlate well with estimates of respiratory disease that incorporates blood gas data (6). RAS is the sum of the whole numbers for oxygen concentrations greater than $25 \%$ (adjusted for altitude at the study site), positive end expiratory pressure $\left(\mathrm{cm} \mathrm{H}_{2} \mathrm{O}\right)$, peak inspiratory pressure $(\mathrm{cm}$ $\mathrm{H}_{2} \mathrm{O}$ ), and mechanical breaths per minute (e.g. a patient on $50 \% \mathrm{O}_{2},[21 / 4]$ pressures, and an intermittent mandatory ventilation rate of 30 would have a RAS of 80 ). Three clinical groups were prospectively defined to reflect the expected patterns of respiratory support during the first week of life. Group I included infants who had an RAS value less than 80 on $\mathrm{d} 2$ and 6. Group II consisted of infants who had an RAS value of more than 80 on $\mathrm{d} 2$ and less than 80 by d 6 , and group III included infants with an RAS value greater than 80 on both days.

Measurements. Cortisol values were measured using the Diagnostic Corporation kit (Los Angeles, CA) with duplicate samples. The intraassay and interassay variabilities for the assay were 5.6 and $7.5 \%$, respectively. Cross-reaction for all naturally occurring adrenal steroids is less than $0.1 \%$. Cortisol values were converted from $\mu \mathrm{g} / \mathrm{dL}$ to $\mathrm{nmol} / \mathrm{L}$ by multiplying by a factor of 27.6. CBG values were measured at the Nichols Institute Reference Laboratories (San Juan Capistrano, CA).

Statistics. All data were analyzed using the SAS (SAS Institute, Inc., Cary, NC) statistical package in the General Clinical Research Center at the University of New Mexico, School of Medicine. The study was designed to evaluate a model of cortisol on postnatal age with gestational age, RAS group, sex, "use of surfactant," and "use of inotropes" as cofactors using analysis of variance and repeated measures analysis of variance. When gestational age was later chosen as the independent variable, postnatal age became a cofactor. Initial evaluation used gestational age as a continuous variable. The relationship between gestational age and cortisol with surfactant or inotropes as cofactors was statistically significant, but the cells necessary for the statistical evaluation became cumbersome. We reviewed the results for likely breakpoints in the data combined with clinical judgment for groupings. We chose three discrete gestational age groups for further evaluation and presentation of results: 1) 24-27 wk gestational age, 2), 28-31 wk, and 3) 32-36 wk.

Pearson's $\chi^{2}$ test and Trend $\chi^{2}$ tests were used to compare the distribution of sex, use of surfactant, and RAS groups within gestational age groups and to compare the distribution of inotrope use and surfactant use within RAS groups.

\section{RESULTS}

Subjects. Table 1 shows the population characteristics of the 178 infants enrolled in the study. Matched cortisol samples in the morning and afternoon on each day resulted in a sample size of 120 for statistical evaluation. The distribution of girls and boys was not significantly different in any gestational age group. There was a significant decrease in surfactant use with increasing gestational age (group 1,78\%; group 2, 60\%; group $3,50 \% ; p=0.017$ ). Infants were distributed in the three RAS groups as shown. The choice of definitions for the RAS groups excluded four infants whose clinical course did not follow the described patterns. The use of inotropes and surfactant was also significantly correlated with RAS group, with the highest use of these therapies in RAS group III (Table 2).

Cortisol pattern. The first model evaluated was cortisol concentrations on postnatal age. We began by evaluating the presence of a diurnal pattern. We used log transformation after initial examination of the paired $t$ test relationship of morning to afternoon absolute cortisol values demonstrated a significant difference but generated a residual plot that suggested lack of a normal distribution. Using $\log _{10}$ values, the data had a normal distribution.

The difference between morning and afternoon cortisol values was more than 0 on $\mathrm{d} 2\left(\mathrm{~F}_{(2,235)}=4.68, p=0.01\right)$. This result suggested that on $\mathrm{d} 2$, cortisol values may be greater in the morning than afternoon. As such, the morning and afternoon values on each day could not be averaged but were studied separately.

We found no significant changes from d 2 to $\mathrm{d} 6$ using either morning or afternoon cortisol values or sex in the model of cortisol concentrations on postnatal age. The cofactors of gestational age, RAS group, and "use of surfactant" each significantly added to the description using afternoon values. Gestational age was the most significant single factor in explaining the variability $\left(\mathrm{F}_{(2,230)}=5.14, p=0.0073\right)$. Therefore, we changed the model to cortisol concentrations on gestational age. The resulting pattern was such that the younger the infant the higher the cortisol value. Postnatal age, RAS group, use of surfactant, and use of inotropes then became cofactors.

The model was designed to analyze the effect of illness on cortisol concentrations using three indicators of illness: use of exogenous surfactant and inotropes as markers of acute illness and RAS groups to define the pattern of illness over the first 6 $\mathrm{d}$ of life. In a model of cortisol on gestational age with surfactant as a cofactor, we found that gestational age $\left(\mathrm{F}_{(2,225)}\right.$ $=6.71, p=0.0012)$ and surfactant $\left(\mathrm{F}_{(2,225)}=6.63, p=0.001\right)$

Table 1. Population characteristics

\begin{tabular}{lccc}
\hline & \multicolumn{3}{c}{ Gestational age group } \\
\cline { 2 - 4 } Characteristics & $1(24-27 \mathrm{wk})$ & $2(28-31 \mathrm{wk})$ & $3(32-35 \mathrm{wk})$ \\
\hline Sample size & 38 & 85 & 55 \\
Sex (girl/boy) & $16 / 22$ & $50 / 35$ & $23 / 32$ \\
RAS group $(n)$ & & & \\
I (89) & 14 & 54 & 21 \\
II (65) & 9 & 23 & 33 \\
III $(24)$ & 15 & 8 & 1 \\
\hline
\end{tabular}


Table 2. Medication use

\begin{tabular}{ccc}
\hline & \multicolumn{2}{c}{ Percentage of total sample } \\
\cline { 2 - 3 } RAS group & Surfactant* & Inotropes $\dagger$ \\
\hline I $(n=89)$ & 45 & 11 \\
II $(n=65)$ & 78 & 58 \\
III $(n=24)$ & 100 & 88 \\
\hline$* p<0.0001\left(\chi^{2}=23.03\right)$. & & \\
$\dagger p<0.001\left(\chi^{2}=16.80\right)$. & &
\end{tabular}

had independent significant effects such that infants treated with surfactant had lower cortisol values than those not receiving this therapy (Fig. 1). Data from afternoon cortisol values on each day were included in the analysis. The use of inotropes as a cofactor resulted in a significant pattern similar to that seen with surfactant $\left(\mathrm{F}_{(2,230)}=4.00, p=0.01\right)$.

Infants who failed to resolve their early lung disease (RAS group III) had significantly lower cortisol values than those found in the other groups of infants $\left(\mathrm{F}_{(2,238)}=6.62, p=\right.$ 0.0016) (Fig. 2).

We found that postnatal age had an interactive effect with gestational age and surfactant $\left(\mathrm{F}_{(2,219)}=4.17, p=0.017\right)$. Using a model that included all infants, postnatal age had no significant main effect separate from that noted for gestational age and use of surfactant. The interactive effect was further studied by examining the cortisol pattern within the three gestational age groupings with the model cortisol on postnatal age with surfactant as a cofactor (Fig. 3). For all three gestational age groups, cortisol values decreased from d 2 to 6 in infants not receiving surfactant, although the changes were not significant. For groups 2 and 3 , cortisol values increased from d 2 to 6 for those infants receiving surfactant, and thus the patterns for those receiving surfactant and those who did not interact across time resulting in a nonsignificant difference. In contrast, in group 1 (24-27 wk), both surfactant-treated and untreated infants decrease their cortisol values from $d 2$ to 6 $\left(\mathrm{F}_{(2,25)}=3.96 p=0.03\right)$.

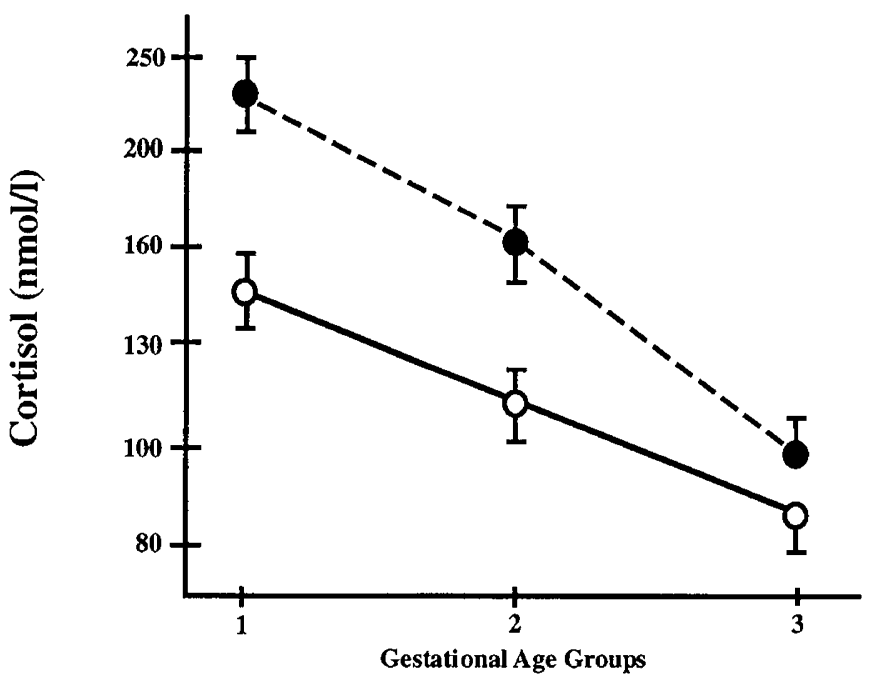

Figure 1. Cross-sectional data for mean \pm SEM cortisol values on gestational age group with surfactant as a cofactor plotted on a semilog graph. Metric units $=$ cortisol $\div 27.6(O$, surfactant treated; $\boldsymbol{O}$, not surfactant treated $)$. $F=6.63$, $p=0.001$.

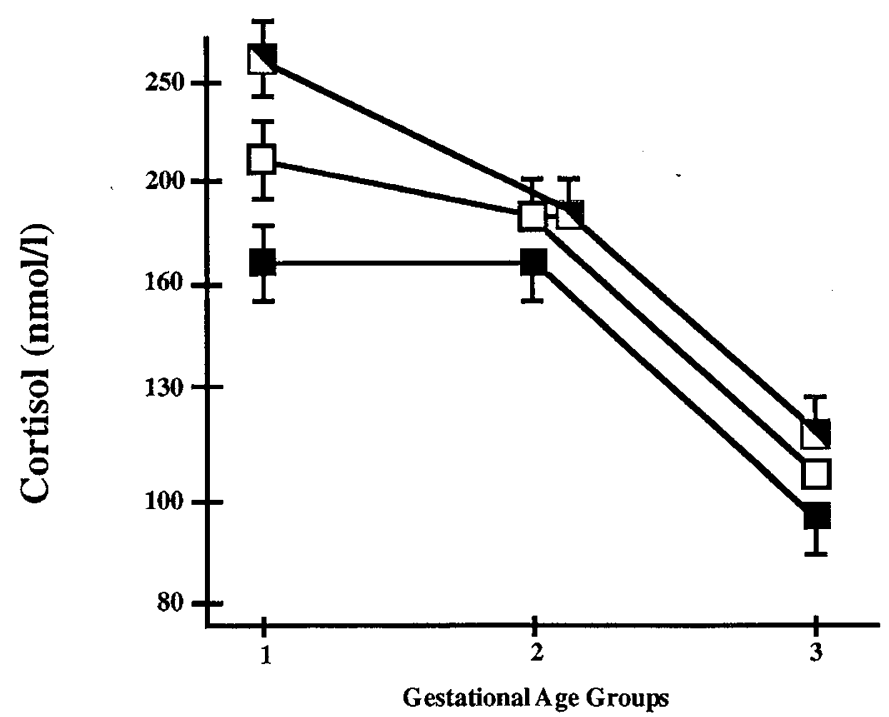

Figure 2. Cross-sectional data for mean \pm SEM cortisol values on gestational age group with RAS group as a cofactor plotted on a semilog graph. Gestational age group $2(\nabla)$ and $1(\square)$ have the same SEM, gestational age group $3(\square)$ is a single subject (RAS groups: $\mathrm{I}=\square, \mathrm{II}=\square$, III $=\square$ ). $F=6.62, p=$ 0.0016 .

$\boldsymbol{C B G}$. There were 61 infants included in the analysis of CBG. The gestational age distribution for this subset was similar to that for the entire group. Mean CBG concentration $(16 \pm 5 \mathrm{mg} / \mathrm{L})$ was less than the adult normal range (19-45 $\mathrm{mg} / \mathrm{L}$ ). There was a trend for younger infants to have lower CBG values, but there was no significant change with gestational age.

\section{DISCUSSION}

We have described the effects of gestational age, postnatal age, and illness on plasma cortisol concentrations in a group of preterm infants. Although previous investigators have reported higher plasma cortisol concentrations in premature infants compared with term infants (7), this is the first study to demonstrate an inverse correlation of gestational age and a concomitant significant negative effect of illness on cortisol values.

We found an inverse relationship between gestational age and cortisol values. The expected higher incidence of illness (higher RAS group) in the more immature infants did not eliminate the gestational age effect, but rather RAS group category was negatively correlated with cortisol values. The net result was that a 24- to 27-wk-old infant had a higher random cortisol value than did the clinically equivalent $32-$ to 36-wk old infant. The physiologic significance of this inverse correlation is unclear. If we postulate that the gestationally older infant has more mature control over baseline cortisol values, then a "high" cortisol value in an immature infant may not necessarily reflect an appropriate response. The recent reports of altered growth hormone (8) and cortisol ultradian (4) patterns in premature infants combined with our results are consistent with a state of immature feedback regulation in the premature infant. An alternative speculation may be that there is decreased tissue sensitivity to cortisol requiring higher cortisol concentrations for normal cortisol action. 


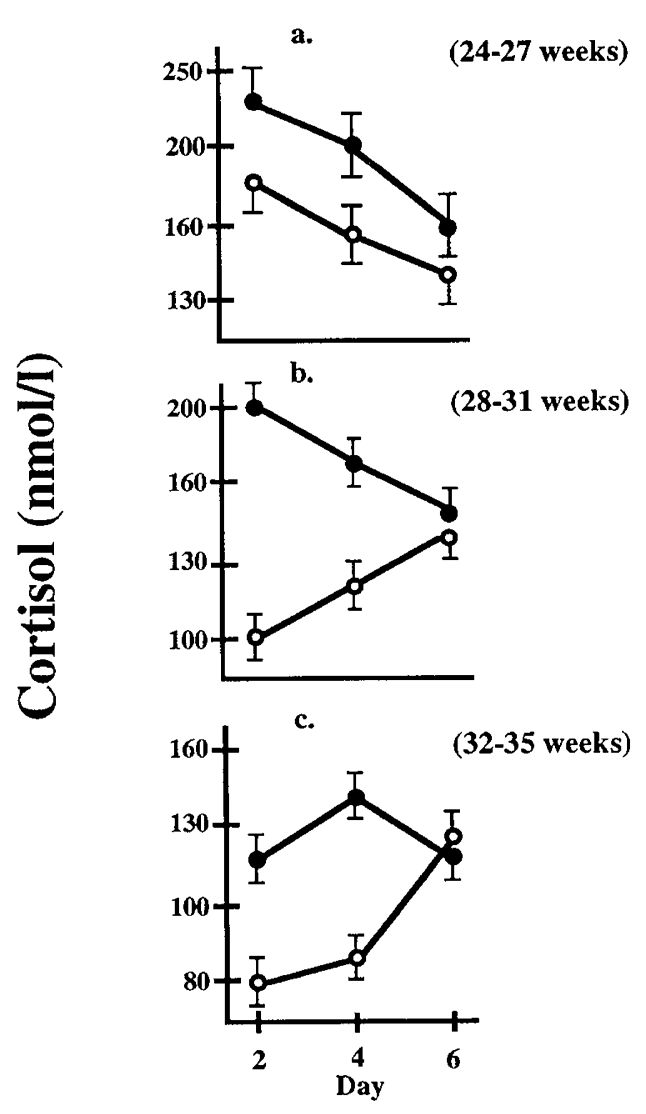

Figure 3. Longitudinal data for mean \pm SEM cortisol values on postnatal age with surfactant as a cofactor plotted on a semilog graph for gestational age group $1(F=3.96, p=0.003)(a)$, gestational age group $2(b)$; and gestational age group $3(c)$. Metric units $=$ cortisol $\div 27.6(\mathrm{O}$, surfactant treated; $\bullet$, not surfactant treated).

The other two markers of illness (use of exogenous surfactant and inotropes) delineated an effect that was also statistically important and independent of the effect of gestational age. Thus, infants who received surfactant (or inotropes) had significantly lower cortisol values compared with the same gestational-aged infants that were well enough to not receive the medications. These factors allowed us to compare the acutely sick versus well infant. Whereas the adult in an intensive care unit has higher cortisol values compared with nonstressed controls (9), this was not the pattern in our study.

The low cortisol values we described may be of clinical significance. Cortisol values were the lowest in those infants who required inotropic support and surfactant, and this relationship was present across gestation. The association of surfactant and inotrope use with lower plasma cortisol values may reflect inadequate cortisol secretion for the production and release of surfactant, and for adequate cardiac function (10).

The lower cortisol values could not be explained by $\mathrm{CBG}$ values. The lower values in premature infants compared with adults were only slightly below the adult range. Furthermore, combining the inverse relationship of cortisol with gestational age and lower $\mathrm{CBG}$ values in younger infants would result in higher free cortisol values in the younger infants.

Our studies were designed to define the postnatal cortisol ontogeny in premature infants. On $\mathrm{d} 2$, there appeared to be a diurnal pattern that was absent after that time. The need for multiple samples to rule out a diurnal pattern means that a significantly lower cortisol value on other days could have been missed. The wide individual variability in cortisol values is consistent with the concept that the magnitude of individual cortisol values after $\mathrm{d} 2$ was probably random and not under mature central feedback regulation.

We found that the effect of postnatal age on cortisol values resulted from an interactive rather than a main effect. This appeared to be due to the heterogeneity of the patient population in its cortisol response. Cortisol values decreased from $\mathrm{d} 2$ to $\mathrm{d} 6$ in all infants who did not receive surfactant and who were thus interpreted as having less significant lung disease. This is similar to the pattern seen in full-term nonstressed infants, in whom the nadir of plasma cortisol is seen at $4 \mathrm{~d}$. In infants who were more than 27 wk gestational age and who received surfactant, cortisol values increased from $\mathrm{d} 2$ to $\mathrm{d} 6$ as would be desired in response to illness. Concomitantly, 68 of 77 infants who received surfactant were resolving their lung disease by d 6 (infants in RAS groups I and II). The resulting intersecting cortisol pattern for well versus ill infants produced a nonsignificant change over time for each gestational age group and showed the "expected" pattern of cortisol values for each subgroup. However, in the youngest gestational age group, cortisol values decreased in infants who received surfactant. Fifty percent (15 of 30) of these infants failed to improve their lung disease by d 6 (RAS group III). Thus, values decreased in both well and sick infants, and the change over time was significant.

In summary, the pattern of cortisol concentrations in the premature infant was significantly correlated with gestational age, with the youngest infants having the highest cortisol values, and inversely correlated with markers of illness. We suggest that the need for surfactant and inotropes and the failure to resolve ventilation needs in premature infants may be due, in part, to clinically inadequate cortisol availability. Gestational age group 1 (24-27 wk) may be at greatest risk. This group of infants had the highest baseline values, which were combined with incidence of significant illness and had a significant decrease in cortisol values across time. By d 6,50\% of the infants were not resolving their lung disease, whereas only $12 \%$ of older infants are not improving. This possibility was further emphasized by the difference in the need for inotropic support within gestational age group 1 when compared by RAS group. Eight percent of infants in RAS I were on inotropic support on 4 or 6 , whereas 22 and $87 \%$ of RAS groups II and III, respectively, were still on inotropic support. We suggest that the decreased efficiency of both production (known to be present in premature infants) and maintenance of high baseline values (or decreased sensitivity at the tissue level) combined with decreasing cortisol values in a setting of continued illness defined a group of infants who expressed a high incidence of cortisol insufficiency. Studies that compare these markers and potential signs of adrenal insufficiency with ACTH-stimulated release of cortisol in a model that includes gestational age will be necessary to further evaluate the clinical significance of our findings. 


\section{REFERENCES}

1. Thomas S, Murphy JF, Dyas J, Ryalls M, Hughes IA 1986 Response to ACTH in the newborn. Arch Dis Child 61:57-60

2. Ward MR, Kimura RE, Rich-Denson CC 1991 Addisonian crisis in extremely premature neonates. Clin Res 37:11A(abstr)

3. Doerr HD, Sippell WG, Versmold HT, Bidlingmaier F, Knorr D 1988 Plasma mineralocorticoids, glucocorticoids, and progestins in premature infants: longitudinal study during the first week of life. Pediatr Res 23:525-529

4. Metzger DL, Wright NM, Veldhuis JD, Rogol AD, Kerrigan JR 1993 Characterization of pulsatile secretion and clearance of plasma cortisol in premature and term neonates using deconvolution analysis. J Clin Endocrinol Metab 77:458-463

5. Hanna CE, Keith LD, Colasurdo MA, Buffkin DC, Laird MR, Mandel SH, Cook DM, LaFranchi SH, Reynolds JW 1993 Hypothalamic pituitary adrenal function in the extremely low birth weight infant. J Clin Endocrinol Metab 76:384-387
6. Watterberg KL, Murphy S, and the Neonatal Cromolyn Study Group: Kelly HW, Katz R, McWilliams B, Werner S, Backstrom C, Angelus P 1992 Failure of cromolyn sodium to reduce the incidence of bronchopulmonary dysplasia: a pilot study. Pediatrics 91:803-806

7. Wittekind CA, Arnold JD, Leslie GI, Luttrell B, Jones MP 1993 Longitudinal study of plasma ACTH and cortisol in very low birth weight infants in the first 8 wk of life. Early Hum Dev 33:191-200

8. Wright NM, Northington FJ, Miller JD, Veldhuis JD, Rogol AD 1992 Elevated growth hormone secretory rate in premature infants: deconvolution analysis of pulsatile growth hormone secretion in the neonate. Pediatr Res 32:286-290

9. Frayn KN 1986 Hormonal control of metabolism in trauma and sepsis. Clin Endocrinol 24:577-599

10. Fisher JH, McCormack F, Park SS, Stelzne, T, Shannon JM, Hofmann T 1991 In vivo regulation of surfactant proteins by glucocorticoids. Am J Respir Cell Molec Biol $5: 63-70$ 